Alarcón R., Fuller D. (2002) Intelligent awareness in support of collaborative virtual work groups. Lecture Notes in Computer Science, 2440, 168-188.

\title{
Intelligent Awareness in Support of Collaborative Virtual Work Groups
}

\author{
Rosa Alarcón and David Fuller \\ Pontifical Catholic University of Chile, Computer Science Department, Vicuña Mackenna \\ 4860, 6904411, Santiago, Chile \\ \{ralarcon, dfuller\}@ing.puc.cl
}

\begin{abstract}
Collaborative Virtual Work Groups (CVWG) are small groups of people that perform tasks, share responsibilities, goals and decisions but are distanced in terms of space, time and cultures, and maintain their connections through technology. Collaborative $\mathrm{S}$ ystems support them enabling a shared environment and informing of its state and changes through a mechanism called Awareness. In this paper we argue that these changes and their consequences can be understood in an ontological work context (we call it "se mantic awareness"). This approach allows us to infer the relevance of the awareness info $r$ mation and control its delivery. To do so, we determine a user context (location, device, preferences), which allows us to infer the user willingness to be exposed to this information. The mechanism is enabled through a Multi -Agent System (MAS), where an agency represents each user's perception and actual context. We also present a case study and discuss the obtained results.
\end{abstract}

\section{Introduction}

Current computer network imp rovement and the possibility of remaining networked, has encouraged the organizations to assign tasks to Collaborative Virtual Work Groups (CVWG). They are small groups of people that perform specific tasks, share decisions, goals and responsibilities, but are distanced in terms of space, time and cultures, and maintain their connections through communication technologies [1], [2]. The main advantage of these groups resides in their flexibility, fast conformation, reorganization and breakup, besides the use of work expertise without incurring in reallocation costs.

They need to react rapidly to changes in their environment and face the uncertainty and distrust imposed by their fragmented environment [3], [4], so they must ove rcome their infrastructure and time differences in order to coordinate actions, maintain formal and spontaneous interactions and keep informed of the project's daily activ ities [5], [6].

Collaborative systems allow them to share software artifacts, objects and self representations enabling a virtual shared environment. The state of this environment 
Alarcón R., Fuller D. (2002) Intelligent awareness in support of collaborative virtual work groups. Lecture Notes in Computer Science, 2440, 168-188.

and its changes are rendered to the user through a mechanism called Awareness, that due to the nature of CVWG, needs to be highly proactive (called passive awareness).

The actual approach consi sts of manifesting the user's interest in being notified automatically of changes in predetermined objects in the environment [7], [8]. But, when the environment is dynamically constructed, those objects are created on the fly, so the notification can even be nonexistent. Also, if there is a temporal distance between the team members, it can fail to reach the user in the appropriate time or can overload him or her if the number of events is too high.

In this paper we propose a semantic approach, in the sens e that every object in the shared environment correspond to a work context ontology ${ }^{1}$, so that when an action attempts to change the object's state, we infer the relevance of the resulting event and its consequences from this ontological context. In additio $\mathrm{n}$, we define a user context that comprises a series of electronic places (logical and physical devices) or contact points as well as a preferences profile.

Both contexts allow us to infer the user willingness to be exposed to awareness i nformation given the actual device interaction capabilities, the user preferences and the relevance of the event.

The awareness mechanism is conceptualized as a Multi -Agent System (MAS). An agent is a software process that controls its own actions (autonomy), perceives its environment (react) and can take the initiative to perform an action (proactively) in order to accomplish tasks on behalf of its user [9], [10], [11]. A MAS system can be seen as a collection of possibly heterogeneous agents.

The proposed MAS constitutes a $\mathrm{n}$ awareness server composed of heterogeneous agents, organized as agencies responsible for delivering user awareness information (one agency per user).

Every agency comprises a pair of intelligent agents. One of them is responsible for estimating the rele vance of events perceived in the shared environment, according to the proposed work context ontology. The other one is $r$ esponsible for scheduling the information delivery according to the designed user context.

Each agent's knowledge is maintained in its own separate knowledge base, so they keep a partial view of the shared environment. Each agent within the agency behaves cooperatively as their actions complement each other.

Agents' communicative interaction follows the conventions described by FIPA 2 ACL (Agent Communication Language) standard. FIPA ACL describes a language in which communicative acts can be expressed; those acts are based on Searle's speech act theory [12] grounded in a logical framework. Agents interchange logical propos itions, actions and objects referencing elements of work and user ontologies.

1 An ontology is an explicit formal specification of the terms in the domain and the relations among them [13].

2 FIPA (Foundation for Intelligent Physical Agents) is a non -profit organization aimed at producing standards for the interoperatio $\mathrm{n}$ of heterogeneous software agents. See 'http: //Www. - fipa.orgh for further details. 
Alarcón R., Fuller D. (2002) Intelligent awareness in support of collaborative virtual work groups. Lecture Notes in Computer Science, 2440, 168-188.

Our aim is to build ubiquitous collaborative applications, in the sense that the awareness mechanism can encounter the user, rendering the shared environment to him or her proactively.

Based on the proposed MAS we implemented an awareness server (AwServer) and developed a case study application: a collaborative web Scheduler (CScheduler).

The ontologies and their main inference criteria are presented in section 2 . In se ction 3, we present the proposed MAS architecture. The MAS implementation and the case study are presented in section 4 . Finally, in section 5 we discuss the results.

\section{Semantic Awareness}

Dourish and Belloti [14] define awareness as "an understanding of the activ ities of others, which provides a context for your own activity". As pointed out by [15]: "awareness is a state of mind of a user .... that it involves the activities of others and that it provides a backdrop ('context') for own activities ... while awareness mech anisms are techniques employed by a system to achieve this state of mind"

Despite there is not a precise definition, we can imply that the objective of the awareness mechanism is to support the maint enance of a shared context by the group members, so they can unde rstand how his or her actions fit into the team's overall goals in order to coordinate their actions [16].

But we can distinguish, at least, two kind of context related to the awareness mechanism: a work context in which the group members' actions are int erpreted and is to some extent common to group members; and a user context that involves the actual state of the user when he or she perceives the awareness information (that is, interacts with the awareness mechanism).

Passive awareness is a strategy to build awareness mechanisms, whose objective is to maintain the user informed of events without requiring him or her to make a conscious effort to do so (i.e. sending notifications through a messa ging system, a mail system or something alike) [14], [17].

Due to the physical and temporal distance, the interactions that occur within a CVWG are mainly asynchronous. In this scenario, passive awareness is best suited to keep the team up-to-date to the shared environment's actual state.

The drawback of this appro ach is that it requires the user to explicit his or her interest in a predetermined object (subscription), a task that the users are not always willing to perform [18]. If a designer predetermines those objects, the mechanism could be too rigid or unable to adapt to the team development.

A naive approach consists of observing every object in the shared environment, notifying any change occurred. The evident inconvenience is an information overload that neither guarantees the awareness information reception (the user can be out of reach) nor its coherent perception (if the mechanism employs different channels).

Additionally, the user context is neither static nor homogeneous. So that shared environment's unexpected situations of use (i.e. delays in document reading due to 
Alarcón R., Fuller D. (2002) Intelligent awareness in support of collaborative virtual work groups. Lecture Notes in Computer Science, 2440, 168-188.

time or technological infrastructure differences) are potential sources of misunderstandings, information loss and casual interaction opportunities lost [19].

The underlying problem consists of determining the relevance of events occurred in the shared environment, and recognizing the context in where they are perceived.

We face the problem by defining a work context ontology and a user context. Work ontology allows us to interpret the events and to define a set of rules in order to derive their relevance and consequences. $U$ ser context allows us to schedule the delivering of awareness information, concerning the events taken place, in terms of user's electronic location and time where the user is willing to perceive the information.

To inf er the user willingness we consider the relevance of the information, the user's preferences and the interaction capabilities of his or her actual electronic location. The next sections present the ontological contexts in detail.

\subsection{Work Context}

Ontology. The Workflow community characterizes work context through an explicit model of business processes. This model comprises tasks, schedules, actors, software tools and information sources and allows to determine the current activities, as well as activities' state, history and related events [20], [21], [22], [23].

This approach has a main drawback because it requires a detailed and a nticipated model of the underlying process that is impossible to obtain from CVGW teams, because they conform a heterogeneous environment and do not follow strictly a sta ndard procedure but act according to their needs and team evolution [24].

Although their contexts are built dynamically, their main components remain the same. According to Haake [25], these components define 3 categories: Content (software tools and shared objects), Processes (activities and their schedules) and People (people and teams). So that, work context would emerge from the dynamic articul ation of these components, that is, the dynamic creation of relationships between them.

Those relationships define the semantics associated with work practice: roles, a uthority and responsibility, available resources, task precedence, etc. [26], [27], [28]. That is, the components and their relationships define a work ontology (Fig. 1). 
Alarcón R., Fuller D. (2002) Intelligent awareness in support of collaborative virtual work groups. Lecture Notes in Computer Science, 2440, 168-188.

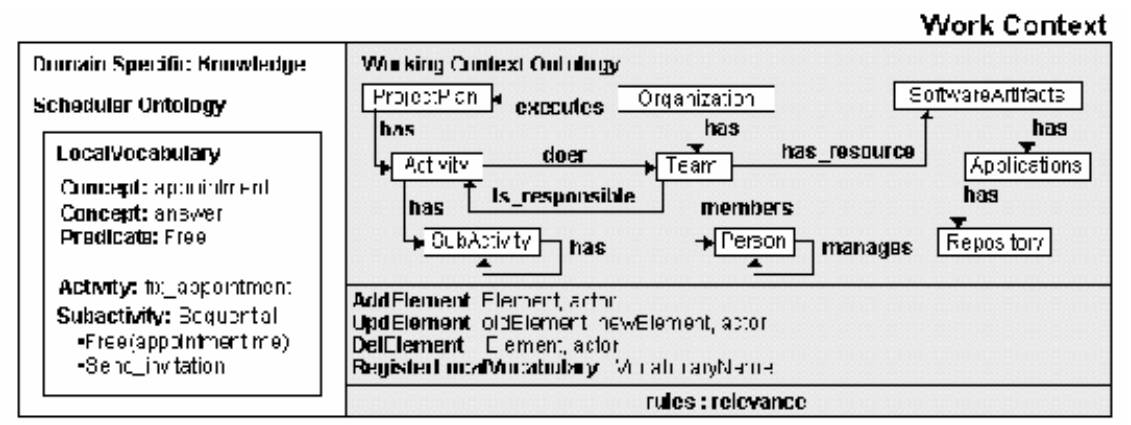

Fig. 1. Content or SoftwareArtifacts component comprises a set of Applications and a generic Repository of shared objects; Process component consists of a ProjectPlan composed of Activities and Subactivities. Person component is implemented via an Organization that includes Teams assigned to accomplish an Activity and Person instances as Team members. The generic operations are also presented (AddElement, UpdElement and DelElement)

Fig. 1 presents the ontology compo nents and their relationships. We also present there the generic operations Add, Update and Delete, and an additional operation called RegisterLocalVocabulary.

This last one represents the operation of extending the ontology vocabulary in o rder to specialize it according to a specific collaborative application.

For example, in the study application (CScheduler), defining a specific activity like fix_appointment requires defining the domain specific concepts "appoin tment", "Free", "answer" (appointment conf irmation), etc., in order to define activity preco nditions and effects or goals (i.e. "Free (me, invitee)"). This is i mportant because a precondition fail must be of interest of the Activity responsible (the team as a whole or a Boss) as well as an Activit y goal achieved. Those are considered logical cons equences of an Activity element (fix_appointment) addition.

Relevance. Events taken place in the shared environment are seen as the result of performing any of the generic operations (Add, Delete, Update) o ver some ontology component instance.

The semantics associated with each operation depends on the component itself (a Delete operation performed over a Person involves a notification sent to the Person itself, but also to the user that makes the call, to t he team where the Person belongs, to his or her Boss, etc.). Those operations are seen as transformation operations [29], executed explicitly or as a logical consequence (implicit) derived from the semantic relationships of the ontology elements.

In relation to human awareness, artifacts have associated transformation operations that are performed when the artifact is used. If an artifact works well (success), it allows us to focus our attention on the main activity objective, thus the artifact is 
Alarcón R., Fuller D. (2002) Intelligent awareness in support of collaborative virtual work groups. Lecture Notes in Computer Science, 2440, 168-188.

transparent to us (its salience is low) until it fails (failure), then it renders itself to us gaining our attention (its salience is high) [29], [30].

According to this theory, we determined some salience or relevance rules considering the result (success or fai lure) of performing any of the envisioned generic oper ations over some instance of an ontology element (Table 1). We take into account the event originator, which is the user that originates the operation (me or a partner); because the transformed artifact exists in the shared environment from which the operation is requested, but also, in each partner's environment.

Table 1. Relevance criteria of events generated when a transforming oper ation is executed considering its result and the pers pective from which it is perceived: the event relevance to me (if I request the operation) and the relevance that it has to my partners (if I request the operation). The scale from minor relevance to a higher one is: none, low, medium, high, sure

\begin{tabular}{llll}
\hline Operation & Result & Relevance to me & Relevance to a partner \\
\hline AddElement & Success & Low & Medium \\
& Failure & High & None \\
UpdElement & Success & Medium & High \\
& Failure & High & Low \\
DelElement & Success & Low & Sure \\
& Failure & Medium & None \\
\hline
\end{tabular}

For example, if a user calls an operation, which is ex ecuted successfully, the event's salience is low. That is to say, the shared environment worked well for the user, it fulfilled his or her expectations. But the salience of the event is higher for his or her partners, because for them the event is unexpected.

\subsection{User context}

E_Place. User context is currently a hot topic of research in the area of context-aware computing [31], [32]. In this community, user context is described in terms of the user's current location taking into account the social - at h ome, at office, etc. (Office Assistant [33], GeoNotes [34]), physical - light, noise, etc. - (Tangible Bits [35]) and informational - guided tours, tourist maps, etc - (CoolTown [36], Tourist Guide [37]) characteristics of the user's current physical environment.

In relation to user location, our focus is on defining electronic places (e_Places), which, resembling abstract contact points, describes the user's location in terms of its interaction capabilities and allow us to reach or contact the user.

Those represent the collection of physical and logical devices available to the user, considered as a whole the awareness mechanism interface [38], [39] (i.e. an e -mail via cellular phone, a collaborative application running in a desktop computer, a pr ivate m essaging system, a WAP system, an SMS system, a beeper, etc.). Fig. 2.A presents some properties that characterizes the class (or concept) e_Place. The fe a- 
Alarcón R., Fuller D. (2002) Intelligent awareness in support of collaborative virtual work groups. Lecture Notes in Computer Science, 2440, 168-188.

tures describe the electronic place in terms of content, location and interaction capabilities.

\begin{tabular}{|c|c|c|c|}
\hline \multirow{3}{*}{ 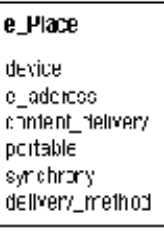 } & \multirow{5}{*}{ (B) } & 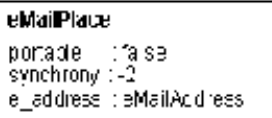 & 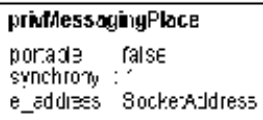 \\
\hline & & WehPlace & 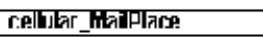 \\
\hline & & 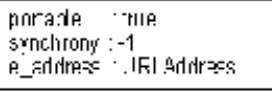 & $\begin{array}{l}\text { porade true } \\
\text { Svichrow : } 2 \\
\text { e_eddress U? LeddJress }\end{array}$ \\
\hline \multirow{2}{*}{ (A) } & & collabAppPlace & cellukal_SWSP|ax \\
\hline & & 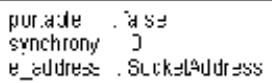 & 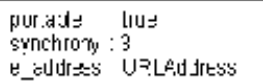 \\
\hline
\end{tabular}

Fig. 2. An $e_{-}$Place (A) is an electronic place that resides in a physical device (a desktop co mputer, a cellular, a PDA, etc.), has an electronic address ( $\quad e \_$address) and a content delivery method. Additionally an $e_{-}$Place is described in terms of its interacti on capabilities, those are: portability, degree of synchrony provided and delivery methods (pull, push or both). We pr esent some e_Places as examples (B), these are: eMailPlace, WebPlace, collabAppPlace, privMessagingPlace, cellular_MailPlace and cellular_SMSPlace

Each e_Place is related to an electronic address (e_Address). Fig. 2 pr esents three examples: an email address (eMailAddress), an URL (URLAddress) and a network address described in terms of socket's host and port (SocketA ddress). These types are arbitrary and could easily be replaced by an URI (Uniform Resource Locator).

For example, the collabAppPlace represents a socket where the collaborative a pplication could listen the awareness mechanism in order to allow the collaborative application's dev eloper to integrate the awareness information in the application interface. The WebPlace represents a Web based collaborative application (a Web site). The cellular_MailPlace and cellular_SMSPlace are associated with web se rvices delivery so they have URLAddresses.

An $e_{-}$Place's content includes its format (string or byte), length and content body (the content itself). An e_Place's location comprises the device where it $\mathrm{r}$ esides (a desktop computer, a cellular, a PDA, etc.), its e_Address, in order to be cont acted; and a method that implements de content delivery through the e_Place.

The $e_{-}$Place's interaction capabilities correspond to the interaction modes that an e_Place affords, and is described by its portability, its deli very method (push, pull or both) and its degree of synchrony.

The degree of synchrony is an index that establishes a measure of potential sy nchrony that could be achieved when an interaction between the awareness mechanism and a user through that e_Place occurs. That is, the greater the index, the shorter the delay between communicative actions. 
Alarcón R., Fuller D. (2002) Intelligent awareness in support of collaborative virtual work groups. Lecture Notes in Computer Science, 2440, 168-188.

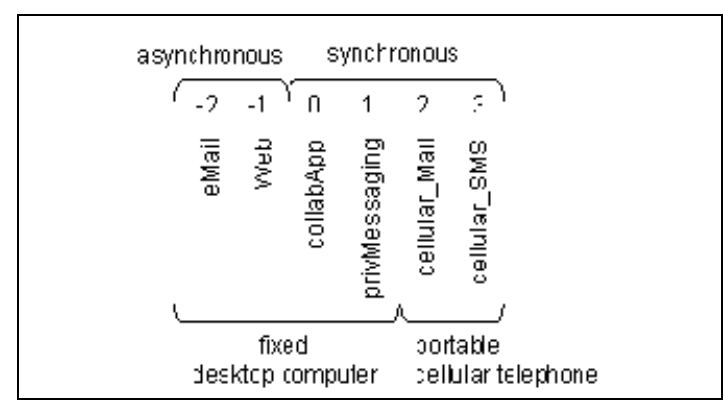

Fig. 3. Examples of E_Places ordered by their synchrony index. E_Places resides in a portable device or in a fixed one. The example devices are a desktop computer and a cellular phone

Fig. 3 presents an e_Places' example ordered according to their synchrony capability. A negative index represents an asynchronous characteristic and a positive index a synchronous one. E_Places residing in portable devices are considered to have a higher synchrony capability than those residing in fixed devices. The presented e_Places resides in a desktop computer and a cellular phone as the figure shows, although the user could have more than one fixed device or portable device at a time.

Fig. 2.B presents some e_P laces as examples. The e_Places in order of synchrony capability are: eMailPlace, WebPlace, collabAppPlace, privMessagingPlace, cellular_MailPlace and cellular_SMSPlace.

Perceptual schedule. E_Places approach, allows us to build ubiquitous collaborative applications, in the sense that the awareness mechanism can encounter the user in some of those e_Places rendering the shared environment to he or she proactively.

The e_Place's inherent interaction capabilities (synchrony) allow us to prescribe an appropriate time and intera ction mode to deliver the awareness information taking into account the relevance of that information in the work context.

We do so via a schedule that relates the information with an appropriate e_Place (location selection) and an appropriate time (time selection) where the information will be perceived (it could be delayed).

Location selection. The appropriate e_Place is inferred considering the relevance of the information in the work context, but also, the e_Place from where the user requested the operation (event source) and his or her current e_Place.

The event's relevance indicates the degree of required synchrony (i.e. associating "urgent" events with e_Places with higher synchrony index).

The event source and the current e_Place describe the coupling level of the interaction (coupled or uncoupled) [24].

That is, if event source and current user location correspond to the same e_Place, then the ongoing interaction is likely to be a coupled one. The selection criterion, in this case, is to direct the awareness information to the event source (Table 2). 
Alarcón R., Fuller D. (2002) Intelligent awareness in support of collaborative virtual work groups. Lecture Notes in Computer Science, 2440, 168-188.

Table 2. E_Place selection criterion for delivering the awareness information assuming that the user has the following e_Places options: "eMail", "Web", "collabApp", "privMessaging", "cellular_Mail" and "cellular_SMS", in increasing disturbance degree order. The criterion takes into account the coupling level of the interaction, the current user location (one of his or her e_Places), the disturbance associated with th e e_Place, and the work relevance (Table 1) to choose an e_Place

\begin{tabular}{lllll}
\hline Relevance & Coupling & $\begin{array}{c}\text { Required in- } \\
\text { teraction }\end{array}$ & Current Location & Delivery \\
\hline low & coupled & synchronous & collabAppPlace & collabAppPlace \\
medium & coupled & synchronous & collabAppPlace & collabAppPlace \\
high & coupled & synchronous & collabAppPlace & collabAppPlace \\
sure & coupled & synchronous & collabAppPlace & privMessagingPlace \\
low & uncoupled & asynchronous & WebPlace & eMailPlace \\
medium & uncoupled & asynchronous & Cellular & eMailPlace \\
high & uncoupled & synchronous & Cellular & cellular_MailPlace \\
sure & uncoupled & asynchronous & Cellular & cellular_SMSPlace \\
\hline
\end{tabular}

An exception is considered for the case related to events with the highest relevance level (sure). In this case, the criterion is to consider the next e_Place in the synchrony order that resi des in the same device, in order to favor coupling interactions (privMessagingPlace in the example).

When the current interaction is uncoupled, the criterion is to direct the awareness information to an asynchronous e_Place. Obviously, selected e_Places mu st have the capability of supporting push technology. WebPlace is the first asynchronous e_Place, but as is a regular Web site, lacks the push capability. For this reason, it is not eligible and the selected e_Place must be the eMailPlace. The exceptions a re those events with higher relevance (high and sure). In these cases, the criterion is to favor synchronous interaction (because they are urgent) so the selected e_Places have the higher synchrony index (cellular_MailPlace and cellular_SMSPlace respectively).

For example, when the user is outside the collaborative application, the e_Place is chosen according to the event relevance ( uncoupled interaction), but when he or she is in the application, the mechanism will prefer the e_Place associated with the co 1laborative application allowing coupled interactions.

When events are the result of inference processes (implicit operations) we assume that the event source is the current user location, in order to favor coupling intera ction.

Time selection. On the othe $r$ hand, the delivery schedule of awareness information must take into account the appropriate time where the information will be perceived.

For synchronous interaction, the chosen time is the closest to the actual (now), while for asynchronous interaction, the time is chosen according to user preferences.

User preferences have been studied in the area of interface agents, indicating that they can be established from the semantics that user actions have in a specific appl ication [9], [10], [39]. 
Alarcón R., Fuller D. (2002) Intelligent awareness in support of collaborative virtual work groups. Lecture Notes in Computer Science, 2440, 168-188.

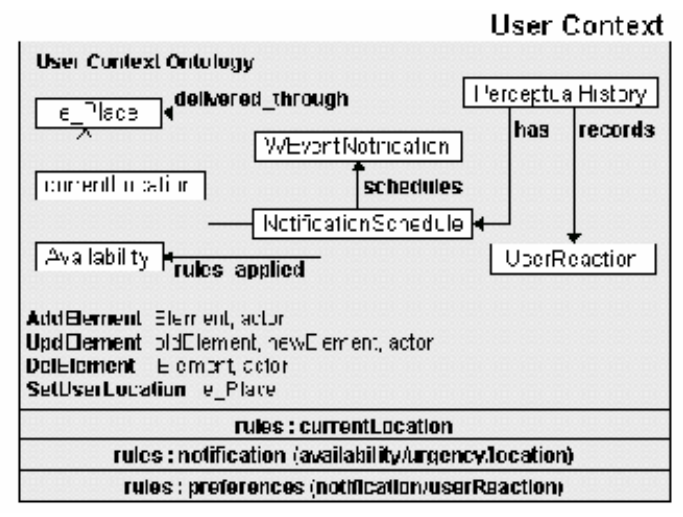

Fig. 4. The WEventNotification is scheduled ( NotificationSchedule), and render through an e_Place, considering its origin, the user currentLocation, his or her Availability patterns, PerceptualHistory (coherence) and UserReaction. The $e_{-}$Place modeling is also presented

The user preferences are recorded in an availability profile based on relevance and time intervals (i.e. "Available (high, 8, 21)"). When an asynchronous interaction is required, notifications are scheduled to the closest available time for the notific ation's relevance (i.e. $8 \mathrm{am}$ if currently is $7 \mathrm{am}$ ). Work events are scheduled for being perceived through e_Places according to availability preferences (Fig.4).

An acknowledgement must be sent indicating that the notifications have reached the user. Otherwise, the notification will increase its urgency level and another e_Place with a higher degree of disruption will be chosen.

When more than one event are scheduled to an e_Place, their precedence order is determined by their arrival order. As the user acknowledges the notification receipt, the event becomes part of the Perceptual History log, and the User Reaction is $r$ ecorded in order to correct or reinforce agents' assumptions.

\section{Architecture}

An agent can be seen as a software proc ess that controls its actions (autonomy), pe rceives its environment (react) and can take the initiative to perform a behavior (proactive) in order to accomplish tasks on behalf of its user.

A MAS can roughly be considered as a system composed of multiple a nd possibly heterogeneous agents. MAS' control can be distributed, so the agents that compose it must communicate among them (social abilities) in order to cooperate and acco mplish their goals [9], [10], [11]. 
Alarcón R., Fuller D. (2002) Intelligent awareness in support of collaborative virtual work groups. Lecture Notes in Computer Science, 2440, 168-188.

Finally, agents require an execution environme nt that put at their reach, the nece ssary sensorial information to perceive their environment (sensor) and act upon it (effector).

In this section we describe the architecture of the proposed awareness mechanism.

Environment. Both work and user context on tologies represent the knowledge of two types of agents, a work and a user context agent, WCA and UCA respectively (See grayed boxes in Fig. 5). A pair of these agents works in a cooperative fashion, conforming an Agency, which is associated to a single user.

The agency is responsible for rendering the awareness information to the user a ccording to the relevance of the information (i nferred for WCA) and the user's current location and preferences (inferred for UCA).

A third agent, called Proxy Agent (PA) has the responsibility to put at the reach of every agency the cap ability of perceiving the shared environment events (sensor) and act upon it (effector).

The collection of agencies conform an Awareness Server (Fig. 5).

In Fig. 5, we present the interactio ns between the main components of the pr oposed architecture. WCA and UCA agents are represented by small heads labeled $W C A$ and $U C A$ respectively, which has above a gray box representing each agent's knowledge.

WCA's knowledge is structured according the wor k context proposed in this paper (Fig. 1); which comprises the general work ontology (labeled as "project know 1edge") as well as a particular instance (labeled "domain specific knowledge") related to a specific collaborative application (the CScheduler in this case).

UCA's knowledge is structured according to the user context proposed which is detailed in Fig. 4.

In both cases we remark the kind of inference rules that apply; for WCA relevance rules are concerned as well as inference rules related to the collaborative applic ation's specific domain. For UCA, the inference rules are related to the determination of the user availability, user location and notification (preferred e_Place and time). 
Alarcón R., Fuller D. (2002) Intelligent awareness in support of collaborative virtual work groups. Lecture Notes in Computer Science, 2440, 168-188.

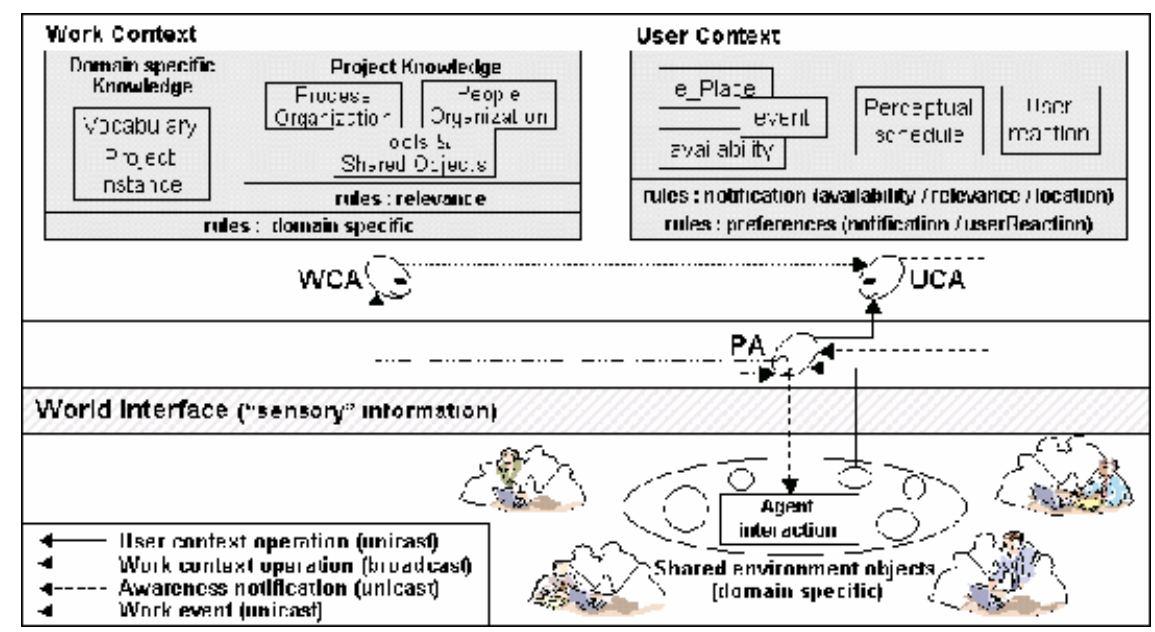

Fig. 5. Every user has an associated agency comprising a WCA agent (Work Context Agent), that infers the relevance of events occurred in the shared environment, and a UCA agent (User Context Agent), that determines the $e_{-}$Place and time where the notification will be delivered. Events are propagated (perceived) to the agency through messages received by a $P A$ agent (Proxy Agent) that broadcasts them to the entire agents' society

When an event occurs in the shared environment, it is propagated to the Agency through the PA agent (an ACL message is sent to the PA agent). If the event is related to work ontology (work context operation), PA broadcasts the message to every WCA agent. If the event is related to user ontology (user context operation), then PA sends the message to the UCA agent that conforms the user's Agency (unicast).

The communication between WCA and UCA (work event) occurs inside the Agency (unicast). User's responses to Agency notifications are also directed to the respective agent (unicast).

The awareness server can be centralized, containing all the age ncies that represent each user. It can also be distributed in local servers containing the user agency, as every agent acts based on its own knowledge base (a partial view of the situation). In both cases, a single PA agent or a collection of them can be i nstanced, but only one agency must be assigned to every user.

Coordination. Agent coordination within an agency is implicit in the sense that each WCA and UCA agents' behavior is designed to complement each other, so they do not need to engage in negotiations in order to fulfill their responsibilities.

Coordination is achieved through message interchange, at knowledge level. Agents' communicative interactions follow social conventions (protocols) defined by the FIPA ACL language, which is based on Searle's speech act theory [12] grounded in a logical framework. Communication occurs as an attempt of informing the agent's 
Alarcón R., Fuller D. (2002) Intelligent awareness in support of collaborative virtual work groups. Lecture Notes in Computer Science, 2440, 168-188.

beliefs, desires or intentions (BDI) state or as an attempt to alter the agent's interloc utors' BDI state.

Messages expressed in FIPA ACL ar e associated with a communicative act that is described in terms of a performative (that is the communicative act type), a specific content and an ontology that is used to interpret the message content.

Messages have precise associated semantics: the conte nt could be logical propos itions, actions or rules referencing elements of work and user ontologies.

Behavior. Communication is directed, and is established between PA and WCA agents (work context operation), PA and UCA agents (user context operation), UCA and PA agents (awareness notification) and WCA and UCA agents (work event) (see Fig. 5 and Fig. 7).

In the first and second cases, the PA agent acts in its role of agency's sensor. That is, PA observes the shared environment and propagates the transformat ion operations occurred to the agency, by requesting WCA and UCA to perform them over the i nstance ontology elements of their respective knowledge bases.

In the third case, PA acts in its role of agency's effector. That is, PA is requested by UCA to effectively notify the user of an occurred event.

Finally, in the fourth case, WCA informs to the UCA agent that the former has i nferred the occurrence of an event in the shared environment as a direct or indirect consequence (inferred) of a transformation operation execution.

So the types of involved communicative acts are directive (requesting an action) and assertive (informing a mental state).

Finally, agents must politely acknowledge their requests for action or information consequences. When an action canno $t$ be accomplished, its failure and possible causes must be informed to the requester. If an agent does not understand a message due to ontology ignorance, it must be informed to the requester.

Knowledge representation. Agents' knowledge related to the devi sed ontologies is maintained in a separate knowledge base (KB). That is a KB for WCA and another for UCA agent.

Facts are asserted in each $\mathrm{KB}$ when a transforming operation is requested trough PA or as a result of each agent's inference processes.

Agents' inference process (relevance and perceptual scheduling) corresponds to a rule-based system, where an inference engine is associated to the agent and its respective KB.

Facts related to the result of performing some transforming operation (events) by the age nt (WCA or UCA) are asserted into the knowledge base. The defined rules derive the consequences of asserting facts (forward chaining).

Agents record or remind their inferences in order to keep the user informed of them. Agents react to their inferences ini tiating a behavior that has as scope the agency (informing their mental) or the awareness mechanism (state or requesting). 
Alarcón R., Fuller D. (2002) Intelligent awareness in support of collaborative virtual work groups. Lecture Notes in Computer Science, 2440, 168-188.

\section{Implementation}

We built an implementation of the proposed MAS system (AwServer) and a collab orative study application (CScheduler). In this section we introduce them.

Both work and user ontology, were developed, as well as a LocalVocabulary that specializes the work ontology according to the semantics of CScheduler. We also built the WCA, UCA and PA agents, their behavior and reasoning mechanism.

\subsection{AwServer}

Tools. AwServer was built over $\mathrm{JADE}^{\mathbf{3}}$, a Java based agent development environment that conforms to FIPA standards, so it provides the services defined by FIPA architecture (see [40] for further detail) as well as some tools to d evelop agents. Fig. 6 presents one of these tools, the sniffer agent, that shows the messages interchanged between agents (right area).

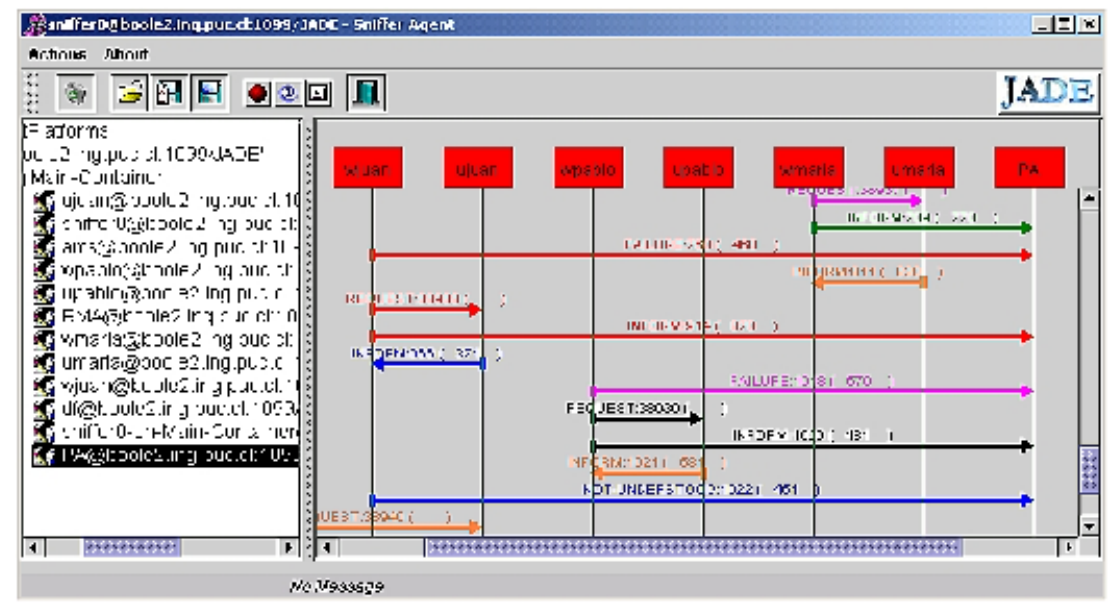

\footnotetext{
${ }^{3}$ Java Agent DEvelopment Framework. See'http://sharon.cselt.it/projects/jadèl.
} 
Alarcón R., Fuller D. (2002) Intelligent awareness in support of collaborative virtual work groups. Lecture Notes in Computer Science, 2440, 168-188.

Fig. 6. Screenshot from JADE's sniffer agent showing the messages interchanged between the $P A$ agent and the agencies of three users: Juan ( wjuan and ujuan agents), Pablo ( wpablo and upablo agents) and Maria ( wmaria and umaria) when attempting to fix an appointment of CScheduler. Top boxes, in the right side represent agents, arrows represent the messages inte rchanged and are labeled with the message's performative

JADE provides the agents' execution platform called an agents' container, which is a JVM (Java Virtual Machine) running in a host computer. Agents developed over JADE are multitasking processes where tasks (or b ehaviors) are executed concu $r$ rently. Message communication is asynchronous and supports some of the FIPA ACL protocols. JADE also support ontology management and mobility.

As FIPA ACL is an abstract content language, it requires an instance language. We chose FIPA SL (semantic language) instance, which defines a syntax message and the REQUEST and INFORM performatives, all supported by JADE.

As the reasoning mechanism we used the Java based inference engine JESS ${ }^{4}$. JESS is an expert system that repeatedly app lies a set of rules to a collection of facts and can interoperate with Java applications.

In order to fulfill their responsibilities, WCA, UCA and PA agents perform a series of behaviors defined in section 3. Below we present the agents' behavior implementation as well as an outline (Fig. 7).

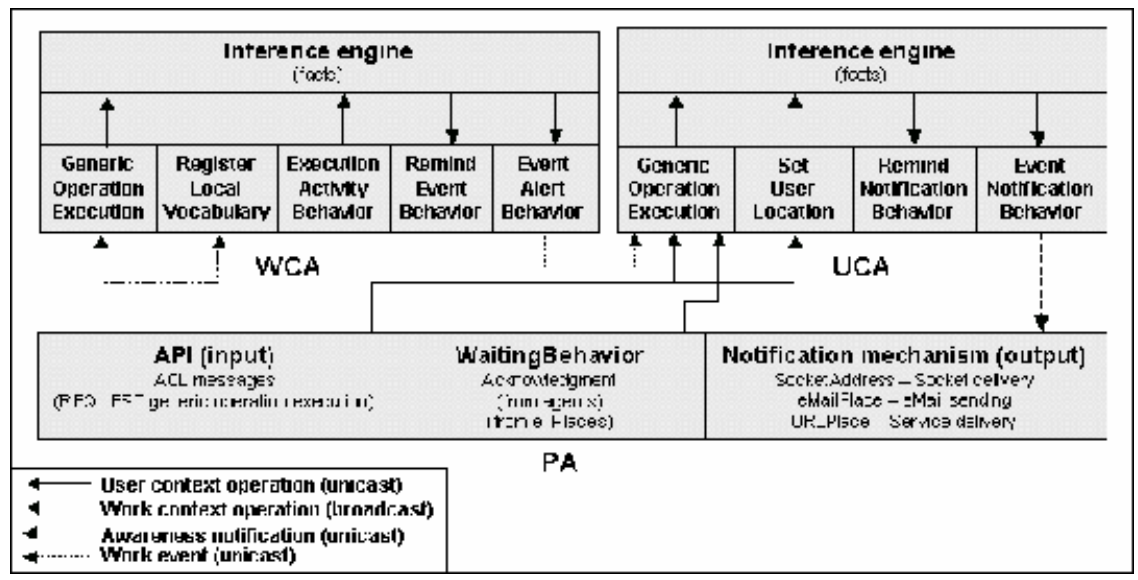

\footnotetext{
${ }^{4}$ Java Expert System Shell. See'http://herzberg.ca.sandia.gov/jess/'
} 
Alarcón R., Fuller D. (2002) Intelligent awareness in support of collaborative virtual work groups. Lecture Notes in Computer Science, 2440, 168-188.

Fig. 7. $P A$ agent acts in its role of sensor ( $A P I$ behavior) and effector (Notification behavior). WCA behavior includes performing transformation operations ( GenericOperationExecution) registering the LocalVocabulary (RegisterLocalVocabulary), executing an Activity ( ExecutionActivity) and informing UCA of an occurred event (EventAlert behavior). UCA actions also include performing transformation operations, as well as keeping the current user location updated (SetUserLocation) and requesting the PA's effector behavior (EventNotification). Both $W C A$ and $U C A$, also reminds the inferred events or notifications

PA behavior implementation. PA agent propagates the changes in the shared environment through an API based on FIPA ACL syntax messages (API behavior).

As agents run in a JVM, that is the entire agents' container is executed as a Java application, the API is implemented as a socket server. PA receives the messages sent from the collaborative application and determines $t$ he appropriate receiver agent type (WCA or UCA agent type). Then PA broadcasts the message to every other agency in the society. The message also includes the user who makes the request (actor) and the e_Place from where is requested.

Since the generic ope rations were developed as part of the ontologies, PA has the capability of "speaking" in both ontologies. API messages performative correspond to FIPA REQUEST.

PA is also responsible for delivering the notification message ( Notification behavior), that is to say, it executes the delivery method (Fig. 2) defined for the e_Place determined for UCA (i.e. open network connections, sending mail, etc.).

Finally, as the notifications must be acknowledged, the PA implements a Waiting behavior, which is an execution suspension until a responding message arrives. This is possible because ACL message has a conversation id field, which is used to reply and build a conversation thread.

WCA behavior implementation. WCA agent receives PA's operation requests and reacts to them initiating a GenericOperationExecution behavior. That is, the agent performs the operation (AddElement, UpdElement or DelELement an element requested) and asserts the resulting action (success/failure) in its own knowledge base.

As the shared environm ent constitutes a domain specific application and is built dynamically, it requires a particular vocabulary and a project instance (i.e. what kind of activities?, what kind of e_Places?). For this reason, the API also implements a RegisterLocalVocabulary behavior.

Also, WCA supports the execution of ontological Activities defined in the LocalVocabulary by describing the activities in terms of logical preconditions and cons equences (ExecutionActivity behavior). The approach allows us to implement conti ngent actions in order to facilitate the collaborative process (the activity execution) as the agents can engage in a FIPA Contract Net protocol to facilitate the preconditions and effects achievement. 
Alarcón R., Fuller D. (2002) Intelligent awareness in support of collaborative virtual work groups. Lecture Notes in Computer Science, 2440, 168-188.

JESS is a reasoning engine that runs repeatedly trying to ap ply the rule set every time that a new fact is asserted. When a rule applies or fires, JESS notifies the event to WCA. The agent records the event (RemindEvent behavior) and sends a message to UCA informing that a new work event has occurred (WEventNotification, see Fig. 4).

UCA behavior implementation. UCA agent also receives PA's operation requests and reacts to them initiating its own GenericOperationExecution behavior. That is, UCA performs the requested operation and asserting the resulting action in $i$ ts own knowledge base.

As the user moves through different e_Places (logging in and out or interacting through them), UCA performs a location updating behavior ( SetUserLocation behavior). That is, updates the user current e_Place.

In a similar fashion to W CA, the JESS engine associated with UCA fires rules $r$ equesting to perform a notification. UCA records the notification to be executed (RemindNotification behavior) and sends a message to PA requesting it to deliver a notification (EventNotification behavior).

Reasoning. As described, both agents implement their reasoning capabilities through JESS. The rules based on criteria depicted on Tables 1 and 2 are defined in the engine when agents are created. Facts are defined by templates or constructs, and rules are applied based on pattern matching techniques.

That is, we defined a series of patterns formed by facts occurrence. For example, if there is an event resulting from an AddElement operation, and the event result is success and I am the operation caller, then the event relevance is low.

When a pattern is matched, then the rules are applied or fired.

\subsection{CScheduler}

The study application is a collaborative scheduler, CScheduler, whose main o bjective is to allow their users to fix appointments between two or more of them.

In order to accomplish this objective, we developed two versions of the sche duler, and associated each of them to two different e_Places for coupled intera ctions. For asynchronous interactions, five e_Places were built.

On the other hand, work ontology was instanced through a LocalVocabulary ( $\mathrm{d}$ omain specific knowledge) which implements basic concepts, predicates and functio nality related to the scheduler.

In this section we describe the e_Places, the work context instantiation and the linkage between AwServer and CScheduler. 
Alarcón R., Fuller D. (2002) Intelligent awareness in support of collaborative virtual work groups. Lecture Notes in Computer Science, 2440, 168-188.

E_Places. We developed two versions of this application, a Java based and a Web based version (Java servlets) and we associated them to two different SocketPlaces. Both of them have associated a SocketAddress (Fig. 2.A) where they can be contacted. So that, when the user is in the shared environment, his or her actions are recognized as a coupled interaction and the awareness information is derived to these channels (Fig. 8.A, 8.B, 8.D).

The other five e_Places were dedi cated to enable asynchronous interactions, those are: an eMailPlace (Fig. 8.C), an additional Soc ketPlace used as a private desktop messaging (Fig. 8.E) and three URLPlaces associated with Web se rvices for message delivery (SMS, cellular mail and ICQ).

For each e_Place, we also implemented the methods associated with the content delivery (Java Mail, Java URL and Java socket invocation).

Domain specific knowledge. As well as user ontology needs to be instantiated through the specification and construction (content delivery methods) of e_Places, work ontology needs to be instantiated through the specification of concepts, predicates and functionality (activities) related to a particular domain, CScheduler in this case.

Work ontology was instanced through a L ocalVocabulary, a class that contains the definitions of basic concepts ("appointment", "answer", etc.) and pred icates ("Free", "Busy") related to the scheduler domain (Fig. 1.). A special remark needs to be done in relation to activity definition (i.e. "fix_appointment").

As we argue in section 2.1 an activity definition involves the definition of its pr econditions ("Free (me, invitee)") and effects or goals ("Busy (me, invitee)"), as well as a start and duration time. So that, if an activity doesn't ful fill its preconditions when startup and hence fails to start, the consequences will be notified automatically to the respo nsible, in our case the user who "creates" the "fix_appointment" activity (the inviter).

Nevertheless the activities are characterized in a particular domain, its conceptual definition allow us to infer logical consequences.

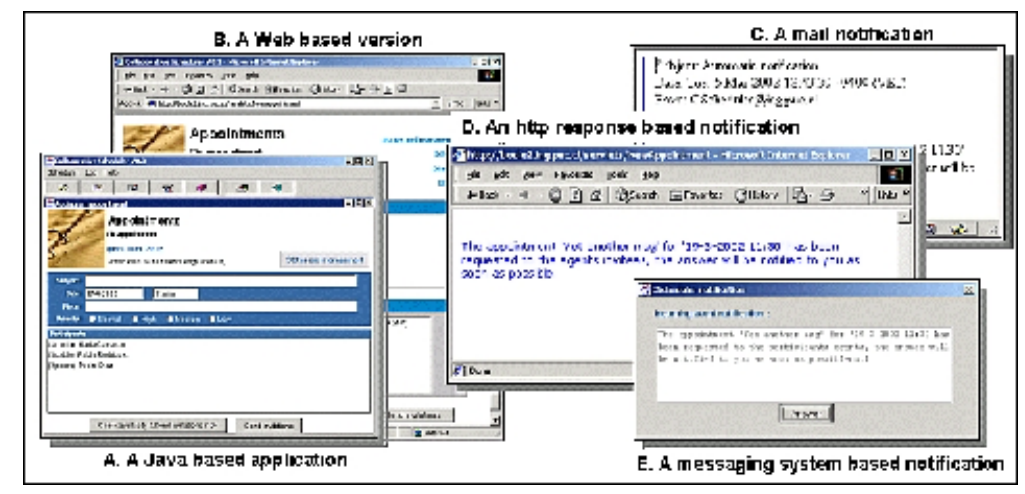


Alarcón R., Fuller D. (2002) Intelligent awareness in support of collaborative virtual work groups. Lecture Notes in Computer Science, 2440, 168-188.

Fig. 8. Screenshots from CScheduler. A) The Java based application version, B) The Web based ve rsion, C) A notification received through the user eMailPlace, D) T he notification through the SocketPlace associated with B) and E) The notification through the Monitor (an independent SocketPlace)

Awareness mechanism. Once the ontologies were instanced, we launched an agency for each user ("juan", "pablo" and "maria"). Actions taken place in the applications (Fig. 8.A, 8.B, 8D, 8E) are propagated to the PA agent by sending ACL messages referred to the ontologies and the generic operations (Add, Delete, Update).

For example, we request to add a "fix_appointment" activity in order to fix an a ppointment. As a result, the agency schedules the activity to start "now", then WCA validates the predicates representing preconditions (i.e. "Free (me, time_interval)" AND "Free (invitee, time_interval)"), if preconditions are satisfie d, it executes the activities' ExecutionBody method and verifies if the effects or goals are achieved (i.e. "Busy (me, time_interval)" AND "Busy (invitee, time_interval)").

As the relationships among an activity, its preconditions, effects and execution (validation, execution and verification) are defined at an abstract level (work onto 1ogy), the JESS engine fires logical cons equences, rate its urgency and determine the e_Place whereto send the event notification.

Fig. 8 presents notifications received when attempting to fix an appointment, through the Monitor (E), an e -mail (C) and the SocketPlace associated with the Web version (an Http response). Fig. 6 presents the exchanged messages in the bac kground between the three agencies representing users "juan", "pablo" and "maria" and the PA agent when attempting to fix an appointment.

\section{Discussion and future work}

The devised architecture enabled us to build a collaborative application with a proa ctive awareness behavior, where the environment can reach the user , taking into a ccount his or her preferences as well as the relevance of the event in the work context.

We believe that the approach would permit the collaborative application developer to implement complex policies from the relevance and availability crit eria, as well as permitting the user to tune those criteria according to his or her actual preferences (they change in time).

However, the chosen knowledge representation does not allow us to d efine stronger restrictions to the relationships between ontol ogy elements (i.e. transitive relationships, numeric quantifiers, etc.), so the logical expressions are not complex enough to allow a latter dynamic context construction.

In addition, the approach demands a large effort from the user to explicit his or her availability preferences and is not able to adapt to changes in work and user co ntext; that is the rules are not inferred or learned. 
Alarcón R., Fuller D. (2002) Intelligent awareness in support of collaborative virtual work groups. Lecture Notes in Computer Science, 2440, 168-188.

Finally, the user context's elements User Reaction and Perceptual History are not currently implemented. These elements a re very important because they will allow the agency to adapt to user preferences dynamically.

In order to accomplish these objectives, our next step consists of employ OIL (O ntology Inference Layer) [41] for a richer ontology representation, as well as to experiment with FACT (Fast Classification of Terminologies) [42] for deriving rules.

FACT is a server that provides infe rence services based on concepts (ontology elements) classification. Concepts must be expressed in OIL, which is based on d escription logics (DL). DL allows the expre ssion of logical propositions in modal and temporal logics.

Based on those changes we plan to include machine -learning techniques to infer relationships between work context elements, and to recognize availability prefe rences in order to allow the agency to adapt to the teams' changes.

Finally we plan to include another agent to the agency in order to create an agency-user interface. This will allow the user and the developer to perform queries and tune the criteria, and the agency to explain its reasoning, but also to receive neg ative feedback from the user in order to adjust the agency's inference processes.

\section{References}

1. Palmer, J., Speier, C.: Teams: Virtualness and media choice. In: International Journal of Electronic Commerce. 3, 1, (1998) 27-48.

2. Lipnack, J., Stamps, J.: Virtual teams: Reaching across space, time, and organizations with technology. John Wiley and Sons, Inc. New York, (eds) (1997).

3. Jarvenpaa, S., Leidner, D.: Communication and Trust in Global Virtual Teams. I n: Journal of Computer-Mediated Communication, Vol.3 (4) (1998).

4. Steinfield, C., Jang, C.: Supporting Virtual Team Collaboration: The TeamSCOPE Sy stem. In: Proc. of GROUP'99. Int. ACM SIGGROUP Conf. on Supporting Group Work (1999).

5. Galegher, J., Kraut, R.: Computer-mediated communication for intellectual teamwork: A field experiment in group writing. In: Proceedings of CSCW'90 (1990) 65-78.

6. Kraut, R., Galegher, J., Egido, C.: Relationships and Tasks in Scientific Research Collaboration. Human Computer Interaction. 3 (1) (1988) 31-58.

7. Leland, M., Fish, R., Kraut, R.: Collaborative Document Production Using Quilt. In Pr oceedings of CSCW 88, (1988) 206-215.

8. Appelt, W.: WWW Based Collaboration with the BSCW System. In Procee dings of SOFSEM'99, Springer Lecture Notes in Computer Science 1725, (1999) 66-78.

9. Maes, P.: Agents that reduce work and information overload. Communications of the ACM, 37 (7) (1994) 31-40.

10.Nwana, H.: Software Agents: An Overview. Knowledge Engineering Review, Vol.11, N.3 (1996) 1-40.

11.Wooldridge, M., Jennings, N. R.: Intelligent Agents: Theory and Practice. In: Knowledge Engineering Review Volume 10 Nro 2. Cambridge University Press (1995).

12.Searle, J. L., Speech Acts. Cambridge University Press (1969).

13.Gruber, T.R.: A translation approach to p ortable ontology specification. Knowledge Acqu isition. Vol. 5 (1993) 199-220. 
Alarcón R., Fuller D. (2002) Intelligent awareness in support of collaborative virtual work groups. Lecture Notes in Computer Science, 2440, 168-188.

14.Dourish, P., Belloti, V.: Awareness and coordination in shared workspaces. In Procee dings of CSCW '92 (1992) 107-114.

15.Sohlenkamp, M.: Supporting Group Awareness in Multi -User Env ironments through Pe rceptualization. GMD Research Series; No. 6. (1999)

16.Fussell, S., Kraut, R., Lerch, F., Scherlis, W., McNally, N., Cadiz, J.: Coordination, overload and team performance: Effects of team communication strategies. In Proceedings of CSCW'98, (1998) 275-284.

17.Gutwin, C., Greenberg, S.: A framework of Awareness for Small Groups in Shared Wor kspace Groupware. Technical Report 99 -1, Department of Computer Science, Unive rsity of Saskatchewan, Canada (1999).

18.Grudin, J.: Groupware and social dynami cs: Eight challenges for developers. Communic ations of the ACM, 37,1 (1994) 92-105.

19.Belloti, V., Edwards, K.: Intelligibility and Accountability: Human Considerations in Co ntext Aware Systems. Human Computer Interaction. Vol. 16. Special Issue on Context Aware Computing (2001).

20.De Michelis, G.: Computer Support for Cooperative Work: Computers Between Users and Social Complexity. In: Zucchermaglio, Bagnara, Stucky (eds.) Organizational Learning and Technological Change, NATO Series, Berlin: Springer Verlag, Vol.141 (1995) 307-330.

21.Agostini, A., De Michelis, G., Grasso, M., Prinz, W., Syri, A.: Contexts, Work Pro cesses and Workspaces. In: CSCW: The Journal of Collaborative Computing. Vol.5, №2 -3 (1996) 223-250.

22.Haake, J., Wang, W.: Flexible Support for Busine ss Processes: Extending Cooper ative Hypermedia with Process Support. In: Information and Software Technology, Vol. 41, No. 6 (1999) 355-366.

23.Carlsen, S.: Conceptual Modeling and Composition of Flexible Workflow Models. PhD Thesis, Information Systems Group, Dep. of Computer and Information Science, Norwegian University of Science and Technology, Trondheim (1997).

24.Schlichter, J., Koch, M., Bürger, M.: Workspace Awareness for Distributed Teams. In Proc. Workshop Coordination Technology for Collaborative Applications, Singapore (1997).

25.Haake, J.: Structural Computing in the Collaborative Work Domain?. In: Reich, S., Ande rson, K., (eds): Open Hypermedia Systems and Structural Computing. Proc. of the 6 Int. OHS Workshop and 2 Int. SC Workshop. Lecture Notes in Co mputer Science, Vol. 1903, Springer-Verlag, Berlin Heidelberg New York (2000) 108-119.

26.Wang, W., Haake, J.: Tailoring Groupware: The Cooperative Hypermedia Approach. In: Special Issue on Tailorable Systems and Cooperative Work in CSCW: the Journal of Coo perative Computing (1999).

27.Wang, W., Rada, R.: Structured Hypertext with Domain Semantics, ACM Transa ctions on Information Systems, Vol. 16, No. 4 (1998) 372-412.

28.Uschold, M., King, M., Moralee, S., Zorgios, Y.: The Enterprise Ontology. In: Uschold, M., and Tate, A., The Knowledge Engineering Review, Vol. 13. Special Issue on Putting O ntologies to Use (1998).

29.Bannon, L., Bødker, S.: Beyond the Interface: Encountering Artifacts in Use. In: Carroll, J.M. (ed.) Designing Interaction: Psychology at the Human-Computer Interface. Ca mbridge University Press. NY. (1991) 227-253.

30.Winograd, T., Flores, C. F.: Understanding computers and cognition: A new found ation for design. Norwood, NJ: Ablex (1986).

31.Dey, A., Abowd, G., Salber, D.: A conceptual framework and a toolkit for supporting the rapid prototyping of context -aware applications. Human Computer Interaction Vol.16. Sp ecial Issue on Context-Aware Computing. (2001). 
Alarcón R., Fuller D. (2002) Intelligent awareness in support of collaborative virtual work groups. Lecture Notes in Computer Science, 2440, 168-188.

32. Yan, H., Selker, T.: Context -aware office assistant. In: Proceedings of the 2000 Int. Confe rence on Intelligent User Interfaces, New Orleans. ACM Press. (2000) 276-279.

33.Schilit, N., Adams, N., Want, R.: Context -aware computing applications. In: Proc. of IEEE Workshop on Mobile Computing Systems and Applications, IEEE Computer Soc iety Press (1994) 85-90.

34.Persson, P.: Social Ubiquitous Computing. In CHI 2001 Building the Ubiquitous Computing User Experience, Position Paper

35.Ishii, H., Ullmer, B.: Tangible Bits: Towards seamless interfaces between pe ople, bits and atoms. In Proceedings of the CHI '97 Conference on Human Factors in Computing Systems. New York, NY: ACM Press. (1997) 234-241

36.Barton, J., Kindberg, T.: The Cooltown Experience. In CHI 2001 Building the Ubiqu itous Computing User Experience, Position Paper

37.Davies, N., Mitchell, K., Cheverest, K., Blair, G., Developing a Context Sensitive Tou rist Guide. In: First Workshop on Human Computer Interaction with Mobile Devices.

38.Lieberman, H., Selker, T.: Out of Context: Computer Systems That Adapt To, and Learn From, Context. IBM Systems Journal 39, No. 3-4 (2000) 617-32.

39.Abowd, G., Mynatt, E.: Charting Past, Present, and Future Research in Ubiquitous Compu ting. In ACM Transactions on Computer-Human Interaction. V.7 N.1 (2000) 29-58.

40.Bellifemine, F., Poggi, A., Rimassa, G.: JADE - A FIPA-compliant agent framewor k. Proceedings of PAAM 99, London (1999) 97-108.

41.Bechhofer, S., Horrocks, I., Patel -Schneider, P., Tessaris, S.: A proposal for a d escription logic interface. In P. Lambrix, A. Borgida, M. Lenzerini, R. Möller, and P. Patel -Schneider, (eds), Proc. of the International Workshop on Description Logics (DL'99) (1999) 33-36.

42.Horrocks, I., FaCT and iFaCT. In P. La mbrix, A. Borgida, M. Lenzerini, R. Möller, and P. Patel-Schneider, (eds), Procee dings of the International Workshop on Description Logics (DL'99) (1999) 133-135. 